\title{
"Arbitrary" criterion for the diagnosis of acute leukemia
}

\author{
Akut lösemi tanısina yönelik "rastgele" kriter
}

\author{
Abbas Hashim Abdulsalam \\ Department of Hematology Unit, Teaching Laboratories, Al-Yarmouk Teaching Hospital, Baghdad, Iraq
}

To the Editor,

According to the WHO classification of acute leukemia, diagnosis is based on an arbitrary cut-off point of $20 \%$ blasts, as the percentage of bone marrow total or non-erythroid cells, or as the percentage of peripheral blood cells. This cut-off point is also currently used in under-resourced laboratories in which the FAB classification is more commonly used.

This cut-off point seems to be universally accepted, and for the time being represents the bestknown criterion for defining acute leukemia; however, "arbitrary" may still precede the criterion due to the follow:

1. This percentage does not represent a specific biological event in the continuum of an increasing blast count, but is merely, to the best to our knowledge, a cut-off point that facilitates relatively clear classification and therapeutic planning. Nonetheless, some high-risk MDS patients with only $10 \%$ bone marrow blasts are actively treated.

2. A significant difference in the blast percentage cut-off point between peripheral blood and bone marrow is well established in MDS; RAEB1 (blast count $<5 \%$ in peripheral blood and $5 \%-9 \%$ in bone marrow) and RAEB-2 (blast count of $5 \%-9 \%$ in peripheral blood and 10\%$19 \%$ in bone marrow). In acute leukemia no such differentiation exits.

3. Morphological findings of pathological "clonal" blasts (type II that contain Auer rods, PseudoChédiak-Higashi or other specific inclusions that are not seen in reactive marrow) refer to the diagnosis of RAEB-2 or AML; here again the arbitrary cut-off point of $20 \%$ blasts results in a specific diagnosis.

4. The original FAB classification was based for many years on the arbitrary cut-off point of $30 \%$ bone marrow blasts and in the past some patients with 20\%-29\% blasts remained stable for months without chemotherapy.

5. Although there is a general consensus concerning the criterion for identifying myeloblasts as agranular (type I) and granular blasts (types II and III), there is some disagreement concerning their definitions, and in practice it may be a matter of convention (subjective method) to differentiate them from the continuum of cells, such as determining whether the cell is a type III blast or a promyelocyte.

6. Blasts refer to myeloblasts, lymphoblasts, monoblasts, promonocytes, and megakaryoblasts. 
7. Diagnosis of AML-M3 and its variant is not related to the percentage of blasts.

8. Cases with $<20 \%$ blast cells may still be diagnosed as acute leukemia if they present with certain recurrent cytogenetic abnormalities, as in AML M4 with inv $(16)$ or $\mathrm{t}(16 ; 16)$ (p13;q22) and AML M2 with $t(8 ; 21)$.

9. The utilization of a $20 \%$ blast threshold is not really an issue in ALL because most patients at the time of diagnosis already have $<50 \%$ blasts. In AML-M0 and M1 this cut-off point is also not useful.

In conclusion: Morphology is the first-line diagnostic criterion for acute leukemia; however, labo- ratory diagnosis of acute leukemia in modern hematologic practice increasingly relies on objective techniques to detect a specific ultrastructural or genetic abnormality. As such, the era of $20 \%$ blasts as a diagnostic criterion for acute leukemia may not stand the time any longer than that of the old FAB group 30\% blasts lower threshold.

\section{Conflict of interest statement}

The authors of this paper have no conflicts of interest, including specific financial interests, relationships, and/or affiliations relevant to the subject matter or materials included. 